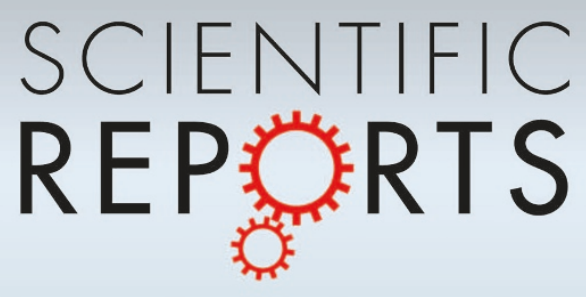

\title{
OPEN \\ Ferroelectric control of a Mott insulator
}

SUBJECT AREAS:

ELECTRONIC DEVICES

FERROELECTRICS AND

MULTIFERROICS

SURFACES, INTERFACES AND

THIN FILMS

ELECTRONIC PROPERTIES AND MATERIALS

Received

8 July 2013

Accepted

9 September 2013

Published

3 October 2013

Correspondence and requests for materials should be addressed to M.B. (manuel.bibes@ thalesgroup.com) or H.Y. (hiroyukiyamada@aist.go.jp)
Hiroyuki Yamada ${ }^{1,2}$, Maya Marinova ${ }^{3}$, Philippe Altuntas ${ }^{2}$, Arnaud Crassous ${ }^{2}$, Laura Bégon-Lours ${ }^{2}$, Stéphane Fusil ${ }^{2,4}$, Eric Jacquet ${ }^{2}$, Vincent Garcia ${ }^{2}$, Karim Bouzehouane ${ }^{2}$, Alexandre Gloter ${ }^{3}$, Javier E. Villegas ${ }^{2}$, Agnès Barthélémy ${ }^{2} \&$ Manuel Bibes $^{2}$

\footnotetext{
${ }^{1}$ National Institute of Advanced Industrial Science and Technology (AIST), Higashi 1-1-1, Tsukuba, Ibaraki 305-8562, Japan, ${ }^{2}$ Unité Mixte de Physique, CNRS-Thales, 1 Av. Augustin Fresnel, Campus de l'Ecole Polytechnique, Palaiseau 911 20, France, and Université Paris-Sud, 91405 Orsay, France, ${ }^{3}$ Laboratoire de Physique des Solides, Université Paris-Sud, CNRS, 91405 Orsay, France, ${ }^{4}$ Université d'Evry-Val d'Essonne, Bd. F. Mitterrand, 91025 Evry, France.
}

The electric field control of functional properties is an important goal in oxide-based electronics. To endow devices with memory, ferroelectric gating is interesting, but usually weak compared to volatile electrolyte gating. Here, we report a very large ferroelectric field-effect in perovskite heterostructures combining the Mott insulator $\mathrm{CaMnO}_{3}$ and the ferroelectric $\mathrm{BiFeO}_{3}$ in its "supertetragonal" phase. Upon polarization reversal of the $\mathrm{BiFeO}_{3}$ gate, the $\mathrm{CaMnO}_{3}$ channel resistance shows a fourfold variation around room temperature, and a tenfold change at $\sim 200 \mathrm{~K}$. This is accompanied by a carrier density modulation exceeding one order of magnitude. We have analyzed the results for various $\mathrm{CaMnO}_{3}$ thicknesses and explain them by the electrostatic doping of the $\mathrm{CaMnO}_{3}$ layer and the presence of a fixed dipole at the $\mathrm{CaMnO}_{3} / \mathrm{BiFeO}_{3}$ interface. Our results suggest the relevance of ferroelectric gates to control orbital- or spin-ordered phases, ubiquitous in Mott systems, and pave the way toward efficient Mott-tronics devices.

T he "Mott transistor", based on the electrostatic carrier-doping of a Mott insulator, has attracted considerable interests as an alternative to the silicon-based field effect transistor (FET), which inevitably confronts a scaling limit ${ }^{1}$. However, a strong modulation of the carrier density is necessary to cause a phase transition in Mott insulators belonging to the strongly correlated oxides family ${ }^{2}$. Although electrolyte-gated FETs satisfy this requirement ${ }^{3}$, they remain impractical for applications. Ferroelectric gating is a promising alternative for solidstate devices ${ }^{4}$, and presents two major advantages: the response time of ferroelectrics is short (in the ns scale or faster), and the induced effects are nonvolatile ${ }^{5-1}$. One of the noteworthy progresses in ferroelectric FETs is the electrical control of functionalities such as superconductivity ${ }^{5-7}$ or ferromagnetism $^{8}$, which occur in correlated oxides. However, less attention has been paid to the potential of ferroelectric field-effects for nonvolatile resistance-switching devices ${ }^{9-12}$.

Most of the previous studies on ferroelectric FETs used as channel material oxide perovskites that originally possessed a high carrier density and therefore a high conductivity ${ }^{9-11}$. However, considering that the FET device we pursue exploits the doping-dependence of the resistivity, $\mathrm{CaMnO}_{3}(\mathrm{CMO})$ is a better suited channel material. This is because $\mathrm{CMO}$ is a Mott insulator that can become metallic upon slight electron-doping, as demonstrated in electrolyte-gating and chemical substitution experiments ${ }^{13}$. For example, as shown in Fig. 1a, in $\mathrm{Ca}_{1-x} \mathrm{Ce}_{x} \mathrm{MnO}_{3}$ (CCMO) 90 unit-cell-thick films a Ce ${ }^{4+}$ concentration as small as $x=0.04$ suffices to induce a metallic character due to electron-doping (Fig. 1b shows a different behavior for thinner films, on which we will come back later).

Another crucial factor for the device performance is the magnitude of the spontaneous polarization in the ferroelectric, and the nature of the structural defects at the interface. Here, we chose "supertetragonal" $\mathrm{BiFeO}_{3}(T-$ $\mathrm{BFO})$ as the ferroelectric gate material. $T$ - $\mathrm{BFO}$ is a polytype of well-known rhombohedral $\mathrm{BFO}(R-\mathrm{BFO})$, which attracts increasing interests due to its huge polarization (possibly $>100 \mu \mathrm{C} / \mathrm{cm}^{2}$ ) at room temperature ${ }^{14-16}$. Furthermore, owing to a giant tetragonal distortion $[a=0.377 \mathrm{~nm}, c=0.465 \mathrm{~nm}$ (ref.15)], T-BFO is almost lattice-matched to CMO having small pseudo-cubic lattice constants $\left(a_{\text {cubic }}=0.373 \mathrm{~nm}\right)$.

On a basis of the above considerations, we fabricated bi-layers composed of CMO and T-BFO, and explored a ferroelectric field effect in these heterostructures.

\section{Results}

Assessments of heterostructures. The T-BFO/CMO heterostructures with $38 \mathrm{~nm}$-thick $T$ - $\mathrm{BFO}$ and various CMO layer thicknesses $\left[6-40\right.$ unit cells (u.c.)] were fabricated onto a $\mathrm{YAlO}_{3}(001)$ substrate $\left(\mathrm{YAO}, a_{\text {cubic }}=0.371 \mathrm{~nm}\right)$. To reduce leakage, $5 \%$ of the $\mathrm{Fe}$ in the $\mathrm{BFO}$ target was substituted by $\mathrm{Mn}^{17}$. Due to the moderate compressive strain, 

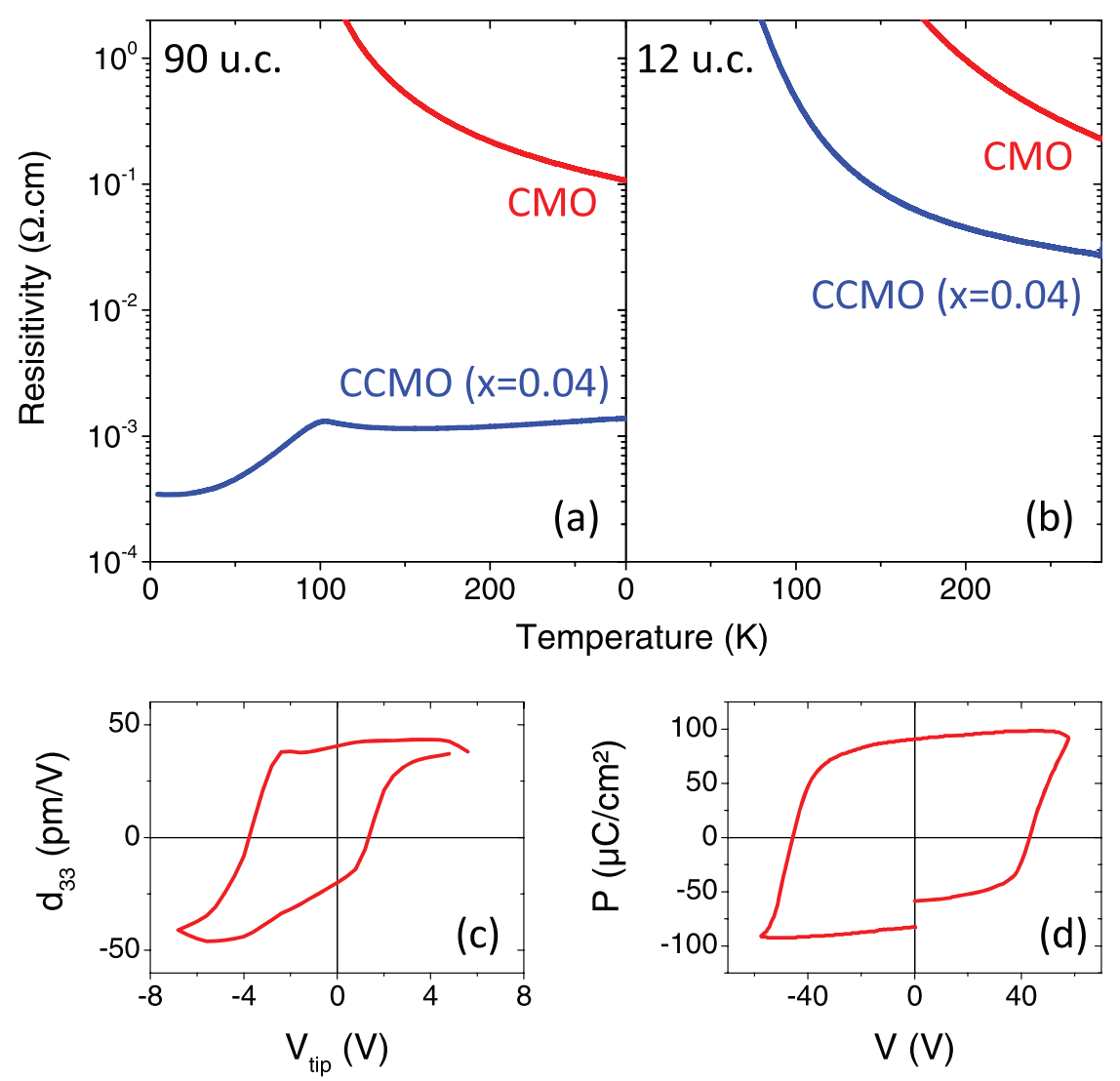

Figure 1 Electronic properties of CMO and T-BFO. (a, b) Resistivity versus temperature for $\mathrm{CaMnO}_{3}\left(\mathrm{CMO}\right.$, red lines) and $\mathrm{Ca}_{0.96} \mathrm{Ce}_{0.04} \mathrm{MnO}_{3}$ $[\mathrm{CCMO}(x=0.04)$, blue lines $]$ films grown on a $\mathrm{YAlO}_{3}(\mathrm{YAO})$ substrate. Thicknesses are (a) 90 unit cells (u.c.) and (b) 12 u.c. (c) Piezoelectric response vs. applied d.c. voltage on AFM tip for the T-BFO/CMO heterostructure evaluated at room temperature. (d) Polarization versus applied voltage measured at the temperature of $77 \mathrm{~K}$ and frequency of $5 \mathrm{kHz}$ for the $120 \mathrm{~nm}$ thick $T$-BFO film with ITO and $\mathrm{CCMO}(x=0.04)$ films as top and bottom electrodes, respectively.

very low electron-doping drives the CMO films into the metallic phase $^{13}$, as evidenced by the properties of CCMO $(x=0.04)$ films grown on YAO (Fig. 1a). As displayed in Fig. 2e, a typical X-ray $2 \theta-\omega$ scan indicates the growth of pure $T$-BFO with a large out-of-plane lattice constant $(0.467 \mathrm{~nm})$, without traces of $R$-BFO.

The characterization of the ferroelectric response of $38 \mathrm{~nm}$ thick $T$-BFO films by standard polarization $v s$. voltage $(P-V)$ measurements proved very challenging due to the significant leakage of this compound. We thus resorted to piezoresponse force microscopy (PFM). This technique is less sensitive to leakage and is based on the mechanical detection of the converse piezoelectric effect that is coupled to ferroelectricity. As visible on the piezoelectric loop of Fig. $1 \mathrm{c}$ the piezoelectric $d_{33}$ coefficient is about $30 \mathrm{pm} / \mathrm{V}$, exhibiting sign reversals when the applied voltage on the tip is smaller than $-4 \mathrm{~V}$ and larger than $+2 \mathrm{~V}$.

On thicker $(120 \mathrm{~nm}) \mathrm{T}$-BFO films [with CCMO $(x=0.04)$ bottom electrode and ex-situ grown $\mathrm{Sn}$-doped $\mathrm{In}_{2} \mathrm{O}_{3}$ (ITO) top electrode pads], we succeeded to measure $P-V$ loops. At room temperature the sample exhibited clear displacement current peaks as a result of polarization reversal, although the significant amount of electric leakage made it difficult to determine $P$ accurately. However, a low-temperature measurement at $77 \mathrm{~K}$ clearly confirms the ferroelectric $P-V$ hysteresis, presented in Fig. $1 d$. Thus, we can claim that the $T$-BFO in the present study possesses a remanent ferroelectric $P$ of about $90 \mu \mathrm{C} / \mathrm{cm}^{2}$, which is larger than typical values obtained for (001)-oriented $R$-type BFO, and consistent with Ref. 16.

The structural details of $T$-BFO/CMO bilayers were further characterized by scanning transmission electron microscopy and electron energy loss spectroscopy (EELS). Fig. 2a shows a high-angle annular dark field (HAADF) image where the contrast mainly arises from atoms with high atomic number ( $\mathrm{Z}$ contrast). The bright spots on the left-hand side correspond to $\mathrm{Bi}$ atoms in the 8 unit-cell thick T-BFO film. The interface with the CMO layer is clearly defined. Below, chemical maps for $\mathrm{Fe}, \mathrm{Ca}$ and $\mathrm{Mn} \mathrm{L}_{3,2}$ edges are presented (Fig. 2b-d). Fe-rich planes are well identified in the BFO film, and individual atomic columns are resolved for $\mathrm{Ca}$ and $\mathrm{Mn}$ in the $\mathrm{CMO}$ layer. Fig. $2 \mathrm{f}$ shows intensity profiles across Fig. $2 \mathrm{a}-\mathrm{d}$, averaged over the rectangular area indicated in Fig. 2a. They allow the identification of the preferential stacking sequence across the $\mathrm{CMO} / \mathrm{BFO}$ interface as $-\mathrm{MnO}_{2}-\mathrm{CaO}-\mathrm{FeO}_{2}-\mathrm{BiO}$ - (is with an A-site electrode termination in the nomenclature of Ref. 18). Because in CMO Mn is $4+$ and $\mathrm{Ca}$ $2+$, while in $\mathrm{BFO}$ both $\mathrm{Bi}$ and $\mathrm{Fe}$ are $3+$, this corresponds to a sheet charge density sequence $0 / 0 /-1 e /+1 e$. This results in a fixed interface dipole pointing toward $\mathrm{CMO}^{18}$. This dipole induces a preference for a downward ferroelectric polarization state, as found experimentally (see the out-of-plane PFM image of Fig. 3b). The shifts $V_{\text {int }}$ toward negative voltage of the $\mathrm{d}_{33}$ loops $\left(\mathrm{V}_{\text {int }} \approx-1.2 \mathrm{~V}\right)$ and $\mathrm{P}(\mathrm{E})$ loop $\left(\mathrm{V}_{\mathrm{int}} \approx-2 \mathrm{~V}\right)$ of Fig. $1 \mathrm{c}$ and $1 \mathrm{~d}$ are consistent with this scenario.

For the FET studies, T-BFO/CMO bilayers were patterned into measurement bridges (see Fig. 3a). In order to reverse the polarization of BFO and pole it "upward" $\left(P_{\text {up }}\right)$ in the channel region between the two voltage legs $\left(\mathrm{V}^{+}, \mathrm{V}^{-}\right.$see Fig. $\left.3 \mathrm{~b}\right)$, we applied $-8 \mathrm{~V}$ while scanning that area. The reversed $P$ is evidenced by a clear phase contrast of 180 degrees in the PFM image, as shown in Fig. 3c, which remains unchanged for at least four weeks. Before and after the poling and PFM reading sequence, we measured the channel resistance underneath the poled region.

Ferroelectric field effect. Figure 4 shows resistivity $v s$. temperature data for CMO channels with $t_{\mathrm{CMO}}=6,10,20$ and 40 u.c. The 

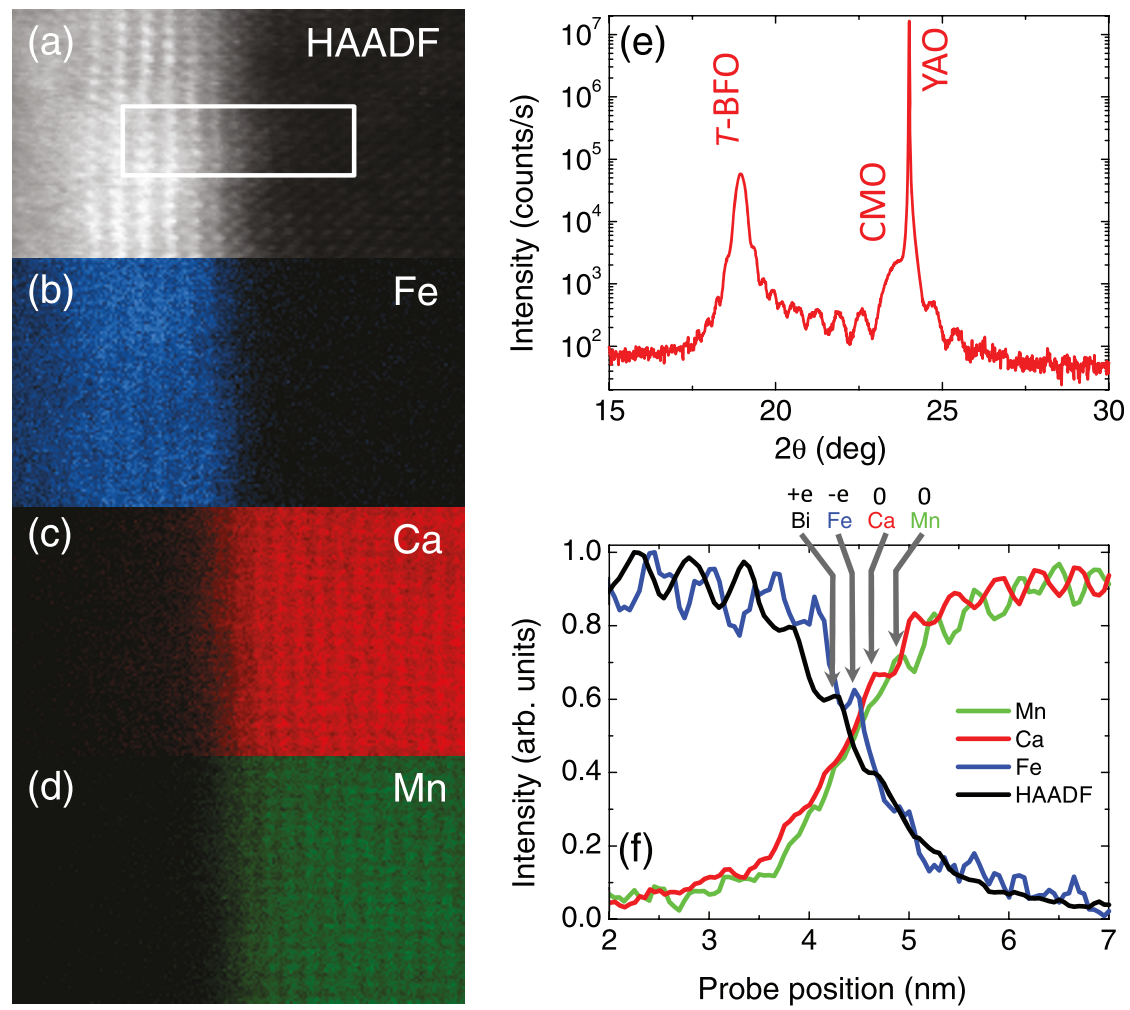

Figure $2 \mid$ Structure properties of T-BFO/CMO heterostructures. (a) High-angle annular dark field image near a T-BFO/CMO interface. (b-d) EELS maps of $\mathrm{Fe}, \mathrm{Ca}$ and $\mathrm{Mn} \mathrm{L}_{3,2}$ edges. (e) X-ray $2 \theta-\omega$ scan of a heterostructure consisting of a $38 \mathrm{~nm}$-thick supertetragonal $\mathrm{BiFeO}_{3}(T$-BFO) and a 40 u.c. CMO layers, grown on YAO. The diffraction peaks are identified as (001) reflections (in pseudocubic setting) of each component. (f) Vertically averaged profiles of the HAADF, Mn, Ca and Fe EELS signals over the rectangular area in (a).

measurements demonstrate a clear correlation between the resistance state and the remanent $P$ in T-BFO: regardless of temperature, the resistivity after poling into $P_{\mathrm{up}}\left(R_{\mathrm{OFF}}\right)$ state is higher than the resistivity in the virgin state $P_{\text {down }}\left(R_{\mathrm{ON}}\right)$ [hereafter, we refer to the high- and low-resistance states as "OFF" and "ON" states, respectively]. The lower resistivity in $P_{\text {down }}$ is consistent with electrostatic electron doping into the channel and the $n$-type conduction in CMO. With positive dc voltage $(+8 \mathrm{~V})$, the OFF state with $P_{\text {up }}$ and $R_{\mathrm{OFF}}$ were switched back to "ON" with $P_{\text {down }}$ and $R_{\mathrm{ON}}$ (Figs. $4 \mathrm{~b}, \mathrm{c}$ ), illustrating

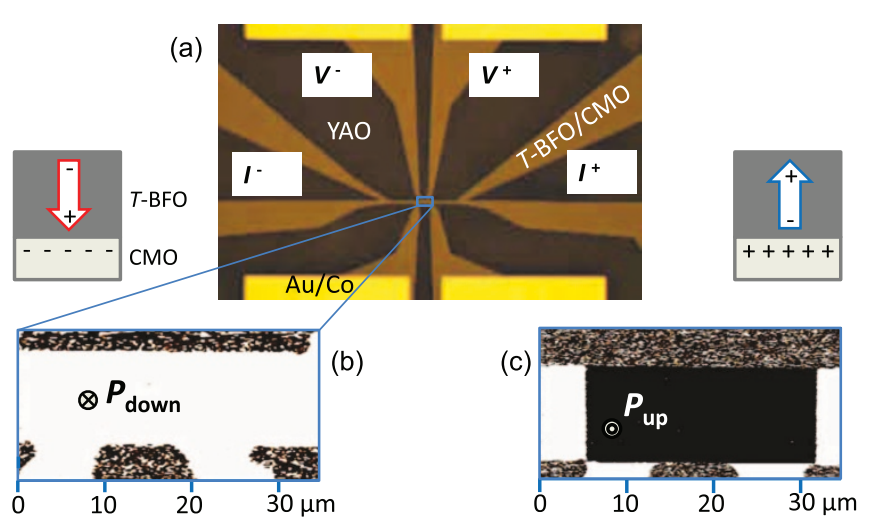

Figure 3 Ferroelectric field effect device. (a) Photograph of a patterned device for PFM and transport experiments. (b) PFM phase image of the active area [white rectangle in (a)] in the as-patterned device, showing a uniform polarization in $T$-BFO pointing toward CMO $\left(P_{\text {down }}\right)$. (c) PFM phase image after writing with $-8 \mathrm{~V}$, demonstrating the reversed polarization $\left(P_{\text {up }}\right)$. the reversibility of the field effect. The sheet resistances in the ON and OFF states are plotted in Fig. 5a-b.

In the device with $t_{\mathrm{CMO}}=20$ u.c. $(=7.5 \mathrm{~nm})$ [Fig. $4 \mathrm{c}$ ], the OFF/ $\mathrm{ON}$ ratio $\left(R_{\mathrm{OFF}} / R_{\mathrm{ON}}\right)$ is nearly 4 at room temperature, and increases as temperature decreases, exceeding 10 at $200 \mathrm{~K}$. In similar devices with e.g. an $\mathrm{La}_{0.8} \mathrm{Sr}_{0.2} \mathrm{MnO}_{3}$ channel and a $\mathrm{Pb}(\mathrm{Zr}, \mathrm{Ti}) \mathrm{O}_{3}$ ferroelectric gate $^{10}, \mathrm{OFF} / \mathrm{ON}$ ratios were found lower than 1.5 at $300 \mathrm{~K}$ and 3 at $200 \mathrm{~K}$. Thus, the present result demonstrates the effectiveness of a channel made of a Mott insulator and of T-BFO for ferroelectric FETs. For $t_{\mathrm{CMO}}=40$ u.c. (Fig. $4 \mathrm{~d}$ ), the OFF/ON ratio decreases by about $40 \%$ as compared to the $t_{\mathrm{CMO}}=20$ u.c. case, see Fig. $5 \mathrm{c}$. Surprisingly, the OFF/ON ratio does not increase beyond its value for 20 u.c. at lower $t_{\mathrm{CMO}}$. This is counter-intuitive, as field-effect is an interface phenomenon and should be enhanced for thinner channels.

To get more insight into the resistive switching mechanism we measured the Hall effect of the devices at $290 \mathrm{~K}$. The Hall coefficient $\left(R_{\mathrm{H}}\right)$ was negative for all measurements. We utilized $-1 / e R_{\mathrm{H}}$ as an estimate of the averaged electron concentration $\left(n_{3 \mathrm{D}}\right)$ that is plotted against $t_{\mathrm{CMO}}$ in Fig. $5 \mathrm{~d}$ (symbols). Here, $e$ represents the elemental charge. $R_{\mathrm{H}}$ for $t_{\mathrm{CMO}}=6$ u.c. could not be evaluated because of the high resistance. If the "native" carrier density in the CMO film were independent of CMO thickness, $n_{3 \mathrm{D}}$ should increase in the ON state $\left(n_{\mathrm{ON}}\right)$ and decrease in the OFF state $\left(n_{\mathrm{OFF}}\right)$ as $t_{\mathrm{CMO}}$ decreases, which qualitatively is what the data displayed on Fig. $5 \mathrm{~d}$ show. However, a closer inspection reveals that the $t_{\mathrm{CMO}}$-dependence of $n_{\mathrm{OFF}}$ is much weaker than that of $n_{\mathrm{ON}}$. In addition, although $\Delta n_{3 \mathrm{D}} \equiv n_{\mathrm{ON}}-n_{\mathrm{OFF}}$ is very large compared to previous results, it should be even higher with a high-polarization ferroelectric such as $T$-BFO. From the observed $P$ $\approx 90 \mu \mathrm{C} / \mathrm{cm}^{2}$ (Fig. 1c), we would expect $\Delta n_{3 \mathrm{D}} \times t_{\mathrm{CMO}}=2 P / e \approx 11$ $\times 10^{14} \mathrm{~cm}^{-2}$. However, for $t_{\mathrm{CMO}}=20$ u.c. with the highest OFF/ON ratio, $\Delta n_{3 \mathrm{D}} \times t_{\mathrm{CMO}}$ is calculated as only $0.6 \times 10^{14} \mathrm{~cm}^{-2}$, corresponding to $2 P \approx 10 \mu \mathrm{C} / \mathrm{cm}^{2}$. 


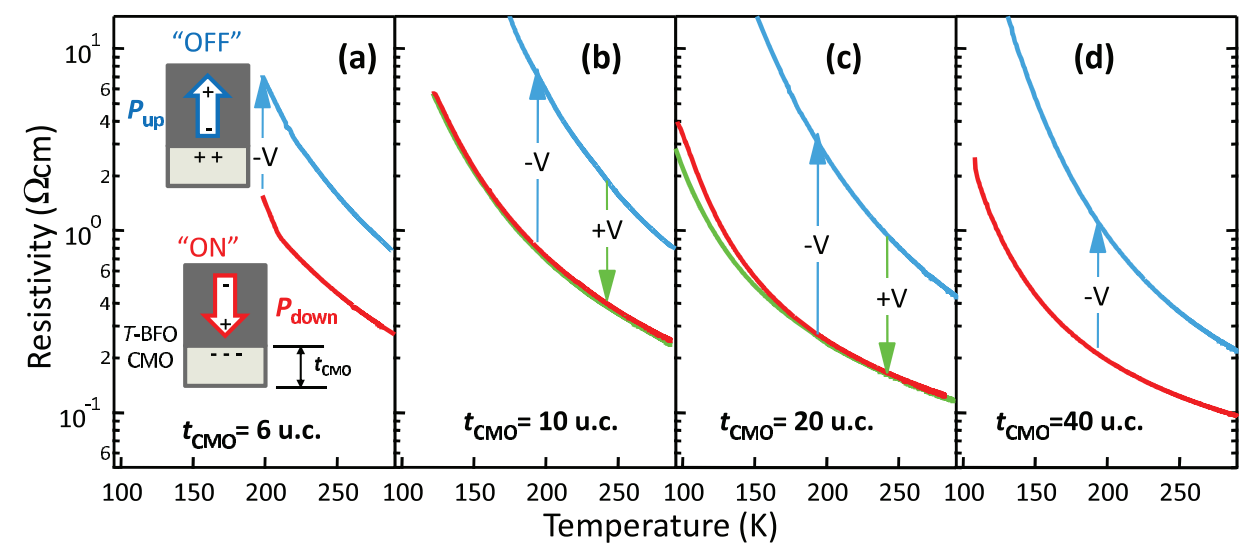

Figure $4 \mid$ Ferroelectric field effect. Resistivity versus temperature for the CMO channel with thicknesses $\left(t_{\mathrm{CMO}}\right)$ of (a) 6 u.c., (b) 10 u.c., (c) 20 u.c., and (d) 40 u.c. The virgin states are shown as red lines ("ON" state), which were switched to "OFF" state (blue lines) with the negative writing voltage ( $-\mathrm{V}$ ), and switched back to "ON" (green lines) with the positive one $(+\mathrm{V})$. Schematics in (a) depict the two states in the device.

\section{Discussion}

The large resistance switching in the $T$-BFO/CMO heterostructure is clearly due to the electrostatic electron-doping of the CMO channel. However, the overall behavior of the devices presents some anomalies. One is related to the asymmetry of the thickness dependence of $n_{\mathrm{OFF}}$ and $n_{\mathrm{ON}}$ (Fig. 5 d). From the STEM/EELS analysis of Fig. 2 we concluded the presence of a fixed interfacial dipole pointing down, which explains the shift of the $d_{33}$ and $P$ vs voltage cycles towards negative voltage (Fig. 1c-d), and favours a downward-oriented polarization state in as-grown samples (Fig. 3b). We argue that this dipole also tends to accumulate charge near the interface between $T$ $\mathrm{BFO}$ and $\mathrm{CMO}$, biasing the ferroelectric-gating effect toward the electron-accumulated state.

A second important feature of our devices is revealed by plotting the sheet conductance $\left(\sigma_{\mathrm{S}}\right)$ at $290 \mathrm{~K}$ as a function of $t_{\mathrm{CMO}}$ (symbols in Fig. 6a). In both the ON and OFF states, the dependence of $\sigma_{\mathrm{S}}$ on the CMO thickness does not extrapolate to zero. Indeed, the conductance vanishes for a CMO thickness around 5 u.c., which suggests the presence of a 5 u.c. "dead layer" in all the devices. The existence of a dead layer in oxide films and devices grown on various different substrates has been largely discussed for hole-doped perovskite manganites $^{19,20}$ and should similarly appear in electron-doped manganites.
In fact, Fig. $1 \mathrm{~b}$ shows that decreasing the CCMO thickness from 90 to 12 u.c. leads to a resistivity increase. Particularly for $x=0.04$, the increase is so strong that the metallic behavior is suppressed, which means that the Ce-doping effect on conductivity is reduced in the ultrathin films. In the phase diagram of the CCMO films ${ }^{13}$, the metallic phase is very narrow because of the strong tendency toward an orbital-ordered phase. Thus, it is natural that the electric conduction in the CMO channel is suppressed if structural disorder or nonstoichiometry exists. The latter are expected at the initial growth stage of $\mathrm{CMO}$ on $\mathrm{YAO}$, and explain the presence of a dead layer at the $\mathrm{CMO} /$ YAO interface.

To gain further insight into the electronic response of our devices we have used a simple electrostatic model. As we discuss below, this model allows us to quantitatively explain the carrier density and conductivity modulation found experimentally, shown in Figs. 5d and $6 \mathrm{a}$. To build this model, we take into account the presence of both a 5 u.c. dead layer and a fixed interfacial dipole, and calculate the sheet carrier density $n_{\mathrm{s}}$ in each perovskite single-plane in CMO, for both directions of the ferroelectric polarization in the T-BFO layer. The range of the carrier accumulation/depletion from the nominal value $n_{s}^{0}=1.1 \times 10^{12} \mathrm{~cm}^{-2}$ is set by the Thomas-Fermi screening length $\lambda$ i.e. $n_{s}-n_{s}^{0} \propto \exp \left(-\frac{t}{\lambda}\right)$ and the free parameters in

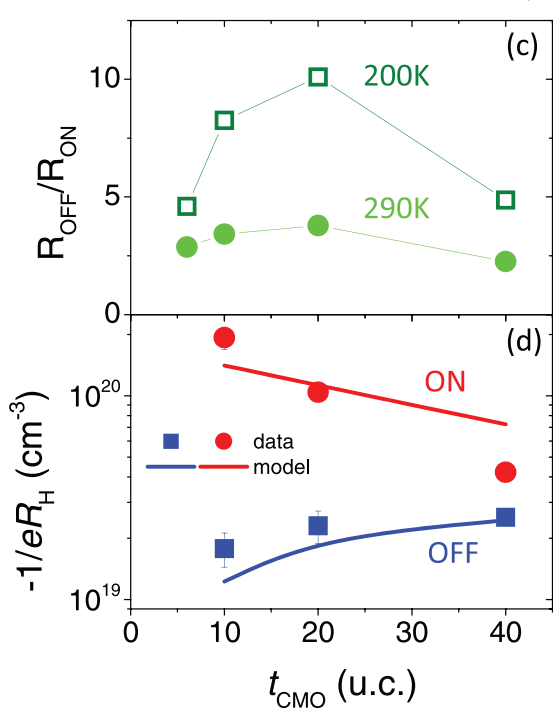

Figure 5 Performance of the CMO/T-BFO ferroelectric FET. Dependence on CMO thickness of the sheet resistance in the ON and OFF states at 200K (a) and $290 \mathrm{~K}$ (b). (c) Resistance ratios between the OFF and ON states compared at $290 \mathrm{~K}$ (squares) and $200 \mathrm{~K}$ (circles), plotted against $t_{\mathrm{CMO}}$. (d) $-1 / e R_{\mathrm{H}}$ plotted against $t_{\mathrm{CMO}}$ for the OFF (blue) and ON (red) states, where $R_{\mathrm{H}}$ and $e$ represent Hall coefficient at $290 \mathrm{~K}$ and elemental charge, respectively. The experimental data are shown as symbols and the lines are simulations with our electrostatic model. 

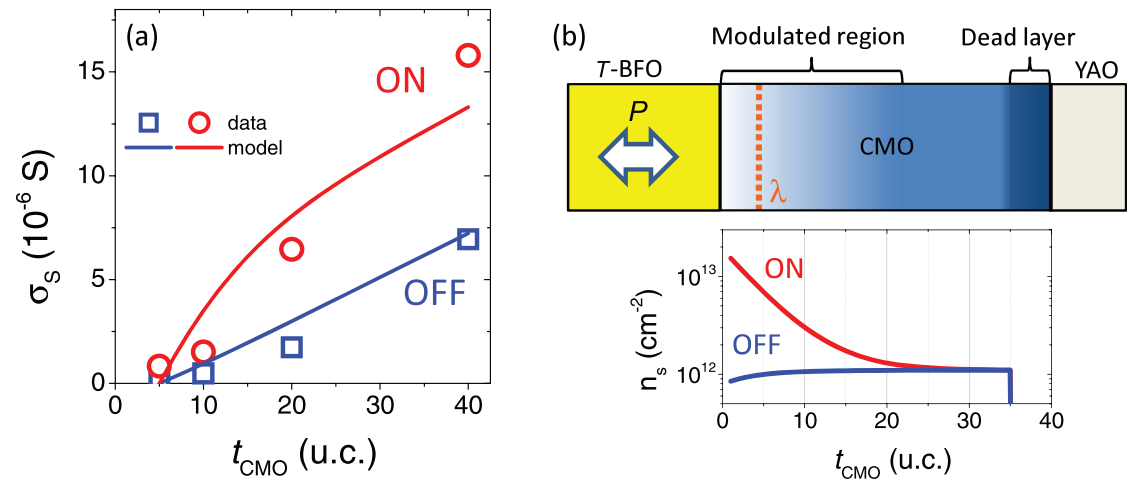

Figure 6 Analysis of the ferroelectric field effect. (a) Sheet conductance $\left(\sigma_{\mathrm{S}}\right)$ at $290 \mathrm{~K}$ in ON (red) and OFF (blue) states plotted against $t_{\mathrm{CMO}}$. The data are shown as symbols and results of the model as solid lines. (b) Depth-profile of the carrier density in the channel. The dotted orange line in the sketch corresponds to the Thomas-Fermi screening length.

the model are $\lambda$ and the amplitude of the fixed interface dipole and of the ferroelectric polarization. Although the absence of conductivity in the dead layer could also be caused by a very low mobility, for simplicity we model it here as a very low concentration of (free) carriers. The graph in Fig. $6 \mathrm{~b}$ shows typical calculated sheet carrier density profiles (the case shown is for $t_{\mathrm{CMO}}=40$ u.c.) for both directions of the ferroelectric polarization. In the $\mathrm{ON}$ state, the polarization points toward the CMO channel and is parallel to the fixed dipole; in the OFF state, it points away from the CMO and is antiparallel to the fixed dipole. $n_{\mathrm{s}}$ is vanishingly small in the 5 u.c. dead layer close to the YAO substrate, weakly affected by ferroelectricity in a "core" region (between about 20 and 35 u.c. where $n_{s}$ remains virtually unchanged at the set value), and strongly dependent on ferroelectric polarization direction in the modulated region (from 0 to about 20 u.c.). The modulation effect, calculated for an effective ferroelectric polarization of $\mathrm{P}=5.5 \mu \mathrm{C} / \mathrm{cm}^{2}\left(2 \mathrm{P}=11 \mu \mathrm{C} / \mathrm{cm}^{2}\right)$, is asymmetric due to the presence of the fixed interface dipole (set to $5 \mu \mathrm{C} / \mathrm{cm}^{2}$, with an uncertainty of $\pm 0.5 \mu \mathrm{C} / \mathrm{cm}^{2}$ ), and extends over more than 20 u.c. In order to fit the data of Fig. 5b, a screening length of 4.5 u.c. was required. This is larger than what can be estimated from the equation $\lambda=\left(\varepsilon \hbar^{2} / 4 m e^{2}\right)^{1 / 2} n^{-1 / 6}(\hbar, m, n, \varepsilon$ are the Planck constant divided by $2 \pi$, the electron mass, the carrier density, and the dielectric constant of CMO, respectively), from which $\lambda=2-3$ u.c. is expected for typical Mott insulator perovskites $\left(n \approx 10^{19} \mathrm{~cm}^{-3}, \varepsilon \approx\right.$ 20). The ferroelectric instability existing in $\mathrm{CMO}$ film $\mathrm{s}^{21}$ or exchange and correlation effects ${ }^{22}$ may however increase $\varepsilon$ and result in a larger $\lambda$. Fig. $6 \mathrm{~b}$ shows that just at the interface, $n_{s}$ is modulated by ferroelectricity over more than one order of magnitude. Such profiles, calculated with the above parameters, yield a good reproduction of the thickness dependence of the carrier density for the ON and OFF states, as visible in Fig. 5 d.

The calculated sheet conductances for the ON and OFF states are plotted vs. CMO thickness in Fig. 6a and compared with the experimental values. This good agreement with the data was obtained by taking into account a carrier mobility of $1.2 \mathrm{~cm}^{2} / \mathrm{Vs}$ at $1.1 \times$ $10^{12} \mathrm{~cm}^{-2}$, slightly decreasing with increasing carrier concentration, in line with what was observed in electrolyte-gated CMO-based transistors ${ }^{13}$. Thus, our simple electrostatic model allows us to quantitatively understand the experimental results.

In summary, we have reported a very large non-volatile modulation of the transport properties in a field-effect transistor combining a Mott-insulator channel and a ferroelectric gate made of supertetragonal $\mathrm{BiFeO}_{3}$. Ferroelectric polarization switching drove resistance and carrier density variations over one order of magnitude. Through a simple electrostatic model taking into account the carrier accumulation/depletion by the ferroelectric polarization and the presence of a fixed dipole, inferred by atomic-scale STEM/ EELS characterization, we have been able to explain the device behavior, for varying channel thickness. Despite the large amplitude of the observed effects, we find that the effective polarization acting on the channel is only about $5 \mu \mathrm{C} / \mathrm{cm}^{2}$, that is much smaller than the polarization of our T-BFO $\left(\sim 90 \mu \mathrm{C} / \mathrm{cm}^{2}\right)$. This may be due to the presence of defects at the channel/gate interface or to the presence of residual nanoscale domains with opposite polarization after poling. Resolving these pending issues to increase the effective polarization by a factor 3-5 should be enough to drive a transition to a metallic state and achieve for the first time a solid-state Mott transistor. For Mott insulators with coupled electronic and magnetic properties, this will also enable the non-volatile electrical control of magnetic and/or orbital order.

\section{Methods}

Fabrication and characterization. The samples were fabricated by pulsed laser deposition using Nd:YAG laser. The substrate temperature $\left(T_{\text {sub }}\right)$ and oxygen pressure $\left(P_{\mathrm{O} 2}\right)$ during the deposition of both CMO and CCMO $(x=0.04)$ films were $670^{\circ} \mathrm{C}$ and $20 \mathrm{~Pa}$, respectively. For the growth of $T-\mathrm{BFO} / \mathrm{CMO} / / \mathrm{YAO}$

heterostructures, the thickness of the CMO layer $\left(t_{\mathrm{CMO}}\right)$ was controlled via reflection high-energy electron diffraction (RHEED). The T-BFO layer was subsequently grown at $T_{\text {sub }}=580^{\circ} \mathrm{C}$ and $P_{\mathrm{O} 2}=0.6 \mathrm{~Pa}$ by using a Mn 5\%-doped target, as described in Ref. 15. Post-deposition annealing was performed at $T_{\text {sub }} \approx 500^{\circ} \mathrm{C}$ and $P_{\mathrm{O} 2}=30 \mathrm{kPa}$.

$2 \theta-\omega$ scans in X-ray diffraction were performed with Panalytical Empyrean equipped with a hybrid monochromator for $\mathrm{Cu} \mathrm{K} \mathrm{K}_{\alpha 1}$ radiation and a PIXcel ${ }^{3 \mathrm{D}}$ detector. The thickness of the T-BFO layer in the heterostructure was measured by X-ray reflectivity with Bruker D8 DISCOVER. The average surface roughness, which can be estimated from fringes in the reflectivity, was cross-checked with atomic force microscopy (AFM), and found as small as $0.4 \mathrm{~nm}$ for all samples.

Measurement bridges for FET studies were patterned by optical lithography and argon ion etching. Electrical contacts for measurements were made on gold/cobalt electrodes defined by a combination of lithography and lift-off techniques.

STEM and EELS. A cross-section of a CMO/T-BFO sample was first prepared by the tripod method for the mechanical polishing and further thinned to electron transparency with a precision ion-polishing system. STEM-HAADF images were acquired using a C3/C5 aberration-corrected STEM, in this case the NION UltraSTEM $100 \mathrm{kV}$ with a probe size of $1 \AA$, allowing an atomic resolution and coupled with EELS experiments performed with a collection semi-angle of $50 \mathrm{mrad}$ and a convergence semi-angle of $35 \mathrm{mrad}$ at a current of approximately $100 \mathrm{pA}$. These experimental parameters enabled us to probe the $3 \mathrm{~d}$ transition metal fine structure (Ca, $\mathrm{Mn}$ and $\mathrm{Fe}_{3,2}$ edges) in the perovskite structure atomic column by atomic column. The EELS spectra were acquired using the spectrum line mode, with an energy dispersion of 0.1 to $1 \mathrm{eV} / \mathrm{channel}$ and an acquisition time from few $\mathrm{ms}$ to $1 \mathrm{~s}$.

PFM measurements. PFM experiments for the $T$-BFO film were conducted with a Digital Instruments Nanoscope IV set-up at room temperature. The piezo-response was detected with commercial silicon tips coated with $\mathrm{Cr}$ and $\mathrm{Pt}$ (Budget Sensors) and external Stanford Research SR830 lock-in amplifiers at an excitation frequency of $4 \mathrm{kHz}$ and an a.c. voltage of $1 \mathrm{~V}$. To pole the channel, a d.c. voltage was applied on the CrPt tip with an internal voltage source in the AFM.

Electrical measurements. The channel resistance was measured by a four-probe configuration with a Keithley 2400 source-measure unit and a Keithley 2182 nanovoltmeter. Its temperature dependence were measured at a constant current of $0.05-0.1 \mu \mathrm{A}$ during a warming run after cooling in a cryostat. For Hall 
measurements, the magnetic field was applied up to $\pm 9 \mathrm{~T}$ in a Quantum Design Physical Properties Measurement System (PPMS). A multifunctional probe in PPMS was utilized for measurements with Keithley 2400 and Keithley 2182. Ferroelectric hysteresis loops for ITO/T-BFO/CCMO films was measured with aixACCT TF analyzer 2000 equipped with a high voltage amplifier. Measurement at $77 \mathrm{~K}$ was carried out in an evacuated probe station cooled with liquid nitrogen.

1. Ahn, C. H. et al. Electrostatic modification of novel materials. Rev. Mod. Phys. 78, 1185-1212 (2006).

2. Imada, M., Fujimori, A. \& Tokura, Y. Metal-insulator transitions. Rev. Mod. Phys. 70, 1039-1263 (1998).

3. Ueno, K. et al. Electric-field-induced superconductivity in an insulator. Nature Mater. 7, 855-8 (2008)

4. Bibes, M. Nanoferronics is a winning combination. Nature Mater. 11, 354-7 (2012).

5. Crassous, A. et al. Nanoscale electrostatic manipulation of magnetic flux quanta in ferroelectric/superconductor $\mathrm{BiFeO}_{3} / \mathrm{YBa}_{2} \mathrm{CuO}_{7-\delta}$ heterostructures. Phys. Rev. Lett. 95, 247002 (2011)

6. Takahashi, K. S. et al. Local switching of two-dimensional superconductivity using the ferroelectric field effect. Nature 441, 195-8 (2006).

7. Ahn, C. H. et al. Electrostatic modulation of superconductivity in ultrathin $\mathrm{GdBa}_{2} \mathrm{Cu}_{3} \mathrm{O}_{7-\mathrm{x}}$ films. Science 284, 1152-1155 (1999).

8. Vaz, C. A. F. et al. Control of magnetism in $\mathrm{Pb}\left(\mathrm{Zr}_{0.2} \mathrm{Ti}_{0.8}\right) \mathrm{O}_{3} / \mathrm{La}_{0.8} \mathrm{Sr}_{0.2} \mathrm{MnO}_{3}$ multiferroic heterostructures. J. Appl. Phys. 109, 07D905 (2011).

9. Ahn, C. H. et al. Local, Nonvolatile Electronic Writing of Epitaxial $\mathrm{Pb}\left(\mathrm{Zr}_{0.52} \mathrm{Ti}_{0.48}\right) \mathrm{O}_{3} / \mathrm{SrRuO}_{3}$ Heterostructures. Science 276, 1100-1103 (1997).

10. Hoffman, J., Hong, X. \& Ahn, C. H. Device performance of ferroelectric/correlated oxide heterostructures for non-volatile memory applications. Nanotechnology $\mathbf{2 2}$ 254014 (2011)

11. Mathews, S., Ramesh, R., Venkatesan, T. \& Benedetto, J. Ferroelectric field effect transistor based on epitaxial perovskite heterostructures. Science 276, 238-40 (1997)

12. Nakano, M. et al. Collective bulk carrier delocalization driven by electrostatic surface charge accumulation. Nature 487, 459-62 (2012).

13. Xiang, P.-H. et al. Strain-mediated phase control and electrolyte-gating of electron-doped manganites. Adv. Mater. 23, 5822-7 (2011).

14. Ricinschi, D., Yun, K.-Y. \& Okuyama, M. A mechanism for the $150 \mu \mathrm{C} \mathrm{cm}^{-2}$ polarization of $\mathrm{BiFeO}_{3}$ films based on first-principles calculations and new structural data. J. Phys.: Condens. Matter 18, L97-L105 (2006).

15. Béa, H. et al. Evidence for Room-Temperature Multiferroicity in a Compound with a Giant Axial Ratio. Phys. Rev. Lett. 102, 217603 (2009).

16. Zhang, J. et al. Microscopic Origin of the Giant Ferroelectric Polarization in Tetragonal-like $\mathrm{BiFeO}_{3}$. Phys. Rev. Lett. 107, 147602 (2011).
17. Zhu, X. H. et al. Thickness-dependent structural and electrical properties of multiferroic $\mathrm{Mn}$-doped $\mathrm{BiFeO}_{3}$ thin films grown epitaxially by pulsed laser deposition. Appl. Phys. Lett. 93, 082902 (2008).

18. Yu, P. et al. Interface control of bulk ferroelectric polarization. Proc. Natl. Acad. Sci. U. S. A. 109, 9710-5 (2012)

19. Tebano, A. et al. Preferential occupation of interface bands in $\mathrm{La}_{2 / 3} \mathrm{Sr}_{1 / 3} \mathrm{MnO}_{3}$ films as seen via angle-resolved photoemission. Phys. Rev. B 82, 214407 (2010)

20. Yamada, H. et al. Engineered Interface of Magnetic Oxides. Science 305, 646-648 (2004).

21. Günter, T. et al. Incipient ferroelectricity in $2.3 \%$ tensile-strained $\mathrm{CaMnO}_{3}$ films. Phys. Rev. B 85, 214120 (2012).

22. Kopp, T. \& Mannhart, J. Calculation of the capacitances of conductors: Perspectives for the optimization of electronic devices. J. Appl. Phys. 106, 064504 (2009).

\section{Acknowledgements}

The authors thank F. Bruno, D. Sando, S. Boyn, N. Reyren, C. Carrétéro, K. March and A. Sawa for useful discussions and experimental helps. This work was supported by the French Agence Nationale de la Recherche NOMILOPS project (ANR-11-BS10-0016), the ERC Advanced Grant FEMMES (contract $n^{\circ} 267579$ ) and $7^{\text {th }}$ framework EU programme ESTEEM2 (grant agreement 312483). H.Y. acknowledges supports from AIST through JSPS “Institutional Program for Young Researcher Overseas Visits", and JSPS KAKENH (No.24760557)

\section{Author contributions}

H.Y., M.B., A.B. and J.E.V. conceived the experiments. H.Y. grew the samples with the assistance of E.J. H.Y., P.A., A.C., S.F., V.G., L.B.L. and K.B. performed the PFM, ferroelectricity and transport measurements. M.M. and A.G. carried out the STEM-EELS characterization. H.Y., J.E.V. and M.B. analysed the results. H.Y. and M.B. wrote the manuscript, with inputs from all other authors.

\section{Additional information}

Competing financial interests: The authors declare no competing financial interests.

How to cite this article: Yamada, H. et al. Ferroelectric control of a Mott insulator. Sci. Rep. 3, 2834; DOI:10.1038/srep02834 (2013).

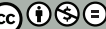

This work is licensed under a Creative Commons Attribution-

NonCommercial-NoDerivs 3.0 Unported license. To view a copy of this license, visit http://creativecommons.org/licenses/by-nc-nd/3.0 